\title{
The viscosity approximation forward-backward splitting method for solving quasi inclusion problems in Banach spaces
}

\author{
Fu Hai Zhao, Li Yang* \\ School of Science, South West University of Science and Technology, Mianyang, Sichuan 621010, P. R. China. \\ Communicated by Y. J. Cho
}

\begin{abstract}
In this paper, we introduce viscosity approximation forward-backward splitting method for an accretive operator and an $\mathrm{m}$-accretive operator in Banach spaces. The strong convergence of this viscosity method is proved under certain assumptions imposed on the sequence of parameters. Applications to the minimization optimization problem and the linear inverse problem are included. The results presented in the paper extend and improve some recent results announced in the current literature. (C) 2017 All rights reserved.
\end{abstract}

Keywords: Accretive operator, viscosity approximation, Banach space, splitting method, forward-backward algorithm. 2010 MSC: 47H09, 47J25.

\section{Introduction}

Splitting methods have recently received much attention due to the fact that many nonlinear problems arising in applied areas such as image recovery, signal processing, and machine learning are mathematically modeled as a nonlinear operator equation and this operator is decomposed as the sum of two (possibly simpler) nonlinear operators. The central problem is to iteratively find a zero of the sum of two monotone operators, namely, let $X$ be a real Banach space, find $x^{*} \in X$ such that

$$
0 \in A x^{*}+B x^{*}
$$

where $A: X \rightarrow X$ is an operator and $B: X \rightarrow 2^{X}$ is a set-valued operator. This problem includes, as special cases, convex programming, variational inequalities, split feasibility problem, linear inverse problem and minimization problem.

A classical method for solving problem (1.1) is the forward-backward splitting method [11, 16, 23, 29] which is defined by the following manner: $x_{1} \in X$ and

$$
x_{n+1}=(I+r B)^{-1}\left(x_{n}-r A x_{n}\right), \quad n \geqslant 1,
$$

\footnotetext{
*Corresponding author

Email addresses: zhaofuhai@swust.edu.cn (Fu Hai Zhao), yangli@swust.edu.cn (Li Yang)
} 
where $r>0$. We see that each step of iterates involves only with $A$ as the forward step and $B$ as the backward step, but not the sum of $B$. This method includes, in particular, the proximal point algorithm $[4,6,14,20,27]$ and the gradient method [3, 13]. Lions-Mercier [16] introduced the following splitting iterative methods in a real Hilbert space:

$$
\left.x_{n+1}=\left(2 J_{r}^{A}-I\right)(2)_{r}^{B}-I\right) x_{n}, n \geqslant 1,
$$

and

$$
x_{n+1}=J_{r}^{A}\left(2 J_{r}^{B}-I\right) x_{n}+\left(I-J_{r}^{B}\right) x_{n}, n \geqslant 1,
$$

where $J_{r}^{T}=(I+r T)^{-1}$. The first one is often called Peaceman-Rachford algorithm [24] and the second one is called Douglas-Rachford algorithm [12]. We note that both algorithms can be weakly convergent in general [23].

In 2012, López et al. [17] introduced the following Halpern-type forward-backward method: $x_{1} \in X$ and

$$
x_{n+1}=\alpha_{n} u+\left(1-\alpha_{n}\right)\left(J_{r_{n}}^{B}\left(x_{n}-r_{n}\left(A x_{n}+a_{n}\right)\right)+b_{n}\right),
$$

where $J_{r}^{B}$ is the resolvent of $B,\left\{r_{n}\right\} \subset(0, \infty),\left\{\alpha_{n}\right\} \subset(0,1]$ and $\left\{a_{n}\right\},\left\{b_{n}\right\}$ are error sequences in $X$. It was proved that the sequence $\left\{x_{n}\right\}$ generated by (1.2) strongly converges to a zero point of the sum of $A$ and $B$ under some appropriate conditions. There have been many works concerning the problem of finding zero points of the sum of two monotone operators (in Hilbert spaces) and accretive operators (in Banach spaces), see $[10,28,29,32]$.

In 2016, Cholamjiak [8] studied a generalized forward-backward method for solving the inclusion problem (1.1) for an accretive and an m-accretive operator in Banach spaces. They then proved its strong convergence under some mild conditions.

The viscosity approximation method for nonexpansive mapping in Hilbert spaces was introduced by Moudafi [22], following the ideas of Attouch [1]. Refinements in Hilbert spaces and extensions to Banach spaces were obtained by $\mathrm{Xu}$ [31].

Let $T: X \rightarrow X$ be a nonexpansive mapping and $f: X \rightarrow X$ be a contraction. Explicit viscosity method for nonexpansive mappings generates a sequence $\left\{x_{n}\right\}$ through the iteration process:

$$
x_{n+1}=\alpha_{n} f\left(x_{n}\right)+\left(I-\alpha_{n}\right) T x_{n}, \quad n \geqslant 0,
$$

where $I$ is the identity of $X$. It is well-known $[22,31]$ that under certain conditions, the sequence $\left\{x_{n}\right\}$ converges in norm to a fixed point $q$ of $T$.

Motivated and inspired by the research going on in this direction. The purpose of this paper is to introduce viscosity approximation forward-backward splitting method for an accretive operator and an $\mathrm{m}$-accretive operator in the framework of Banach spaces. More precisely, we consider the following iterative algorithm:

$$
x_{n+1}=\alpha_{n} f\left(x_{n}\right)+\lambda_{n} x_{n}+\delta_{n} J_{r_{n}}^{B}\left(x_{n}-r_{n} A x_{n}\right)+e_{n}, \quad n \geqslant 1 .
$$

Under certain assumptions imposed on the sequence of parameters, the strong convergence of this viscosity method is proved.

\section{Preliminaries}

Throughout the paper, $X$ is a real Banach space with norm $\|\cdot\|$ and dual space $X^{*}$. The expressions $x_{n} \rightarrow x$ and $x_{n} \rightarrow x$ denote the strong and weak convergence of the sequence $\left\{x_{n}\right\}$, respectively.

The modulus of convexity of $X$ is the function $\delta(\epsilon):(0,2] \rightarrow[0,1]$ defined by

$$
\delta(\epsilon)=\inf \left\{1-\frac{\|x+y\|}{2}:\|x\| \leqslant 1,\|y\| \leqslant 1,\|x-y\| \geqslant \epsilon\right\} .
$$


A Banach space $X$ is said to be uniformly convex, if $\delta(\epsilon)>0$ for every $\epsilon \in(0,2]$.

Let $\rho:[0, \infty) \rightarrow[0, \infty)$ be the modulus of smoothness of $X$ defined by

$$
\rho(t)=\sup \left\{\frac{1}{2}(\|x+t y\|+\|x-t y\|)-1: x, y \in X,\|x\|=\|y\|=1\right\} .
$$

A Banach space $X$ is said to be uniformly smooth if $\frac{\rho(t)}{t} \rightarrow 0$ as $t \rightarrow 0$. Let $q$ be a fixed real number with $q>1$. Then a Banach space $E$ is said to be q-uniformly smooth if there exists a constant $b>0$ such that $\rho(t) \leqslant b t^{q}$ for all $t>0$. It is well-known that every q-uniformly smooth Banach space is uniformly smooth.

Let $\mathrm{J}_{\mathrm{q}}(\mathrm{q}>1)$ denote the generalized duality mapping from $X$ into $2^{\mathrm{X}^{*}}$ given by

$$
\mathrm{J}_{\mathbf{q}}(x)=\left\{j_{\mathbf{q}}(x) \in X^{*}:\left\langle x, j_{\mathbf{q}}(x)\right\rangle=\|x\|^{\mathbf{q}},\left\|j_{\mathbf{q}}(x)\right\|=\|x\|^{\mathbf{q}-1}\right\}, \quad \forall x \in X,
$$

where $\langle\cdot, \cdot\rangle$ denotes the duality pairing between $X$ and $X^{*}$. In particular, $\mathrm{J}_{2}:=\mathrm{J}$ is called the normalized duality mapping on $X$. It is also known (e.g., [30, p.1128]) that

$$
j_{q}(x)=\|x\|^{q-2} J(x), \quad x \neq 0 .
$$

We next provide some properties of the duality mapping.

Lemma 2.1 ([9]). Let $1<\mathrm{q}<\infty$.

(i) The Banach space $\mathrm{X}$ is smooth, if and only if the duality mapping $\mathrm{J}_{\mathrm{q}}$ is single-valued.

(ii) The Banach space $\mathrm{X}$ is uniformly smooth, if and only if the duality mapping $\mathrm{J}_{\mathrm{q}}$ is single-valued and norm-tonorm uniformly continuous on bounded subsets of $X$.

By using the concept of sub-differentials, we know the following inequality:

Lemma 2.2 ([7, p. 33]). Let $\mathrm{q}>1$ and $\mathrm{X}$ be a real normed space with the generalized duality mapping $\mathrm{J}_{\mathrm{q}}$. Then, for any $x, y \in X$, we have

$$
\|x+y\|^{q} \leqslant\|x\|^{q}+q\left\langle y, j_{q}(x+y)\right\rangle,
$$

for all $\mathrm{j}_{\mathrm{q}}(x+y) \in \mathrm{J}_{\mathrm{q}}(x+y)$.

We define the domain and the range of an operator $A: X \rightarrow 2^{X}$ by $D(A)=\{x \in X: A x \neq \emptyset\}$ and $R(A)=\bigcup\{A z: z \in D(A)\}$, respectively. The inverse of $A$, denoted by $A^{-1}$, is defined by $x \in A^{-1} y$, if and only if $y \in A x$. A set-valued operator $A$ is said to be accretive, if for each $x, y \in D(A)$, there exists $j(x-y) \in J(x-y)$ such that

$$
\langle u-v, j(x-y)\rangle \geqslant 0, u \in A x, \quad v \in A y .
$$

An accretive operator $A$ is said to be $m$-accretive, if $R(I+r A)=X$ for all $r>0$.

Given $\alpha>0$ and $q \in(1, \infty)$, we say that an accretive operator $A$ is $\beta$-inverse strongly accretive ( $\beta$-isa) of order $q$, if for each $x, y \in D(A)$, there exists $j_{q}(x-y) \in J(x-y)$ such that

$$
\left\langle u-v, j_{q}(x-y)\right\rangle \geqslant \beta\|u-v\|^{q}, u \in A x, v \in A y .
$$

Let $C$ be a nonempty subset of a real Banach space $X$. Let $T: C \rightarrow C$ be a nonlinear mapping. We denote the fixed point set of $T$ by $\operatorname{Fix}(T)$, that is, $\operatorname{Fix}(T)=\{x \in C: x=T x\}$.

Lemma 2.3 ([25, Corollary 1]). Let $C$ be a closed convex subset of a uniformly smooth Banach space $X$ and let $\mathrm{T}: \mathrm{C} \rightarrow \mathrm{C}$ be a nonexpansive mapping with a fixed point. For each fixed $\mathrm{u} \in \mathrm{C}$ and every $\mathrm{t} \in(0,1)$, the unique fixed point $\mathrm{x}_{\mathrm{t}} \in \mathrm{C}$ of the contraction $\mathrm{C} \ni \mathrm{x} \mapsto \mathrm{tu}+(1-\mathrm{t}) \mathrm{T} x$ converges strongly as $\mathrm{t} \rightarrow 0$ to a fixed point of $\mathrm{T}$.

In what follows, we shall use the following notation:

$$
\mathrm{T}_{r}^{\mathrm{A}, \mathrm{B}}=\mathrm{J}_{\mathrm{r}}^{\mathrm{B}}(\mathrm{I}-\mathrm{rA})=(\mathrm{I}+\mathrm{rB})^{-1}(\mathrm{I}-\mathrm{rA}), \quad r>0 .
$$


Lemma 2.4 ([17, Lemma 3.1 and Lemma 3.2]). Let $X$ be a Banach space. Let $A: X \rightarrow X$ be an $\alpha$-isa of order $\mathrm{q}$ and $\mathrm{B}: \mathrm{X} \rightarrow 2^{\mathrm{X}}$ an m-accretive operator. Then we have

(i) For $r>0$, Fix $\left(T_{r}^{A, B}\right)=(A+B)^{-1}(0)$.

(ii) For $0<\mathrm{s} \leqslant \mathrm{r}$ and $\mathrm{x} \in \mathrm{X},\left\|\mathrm{x}-\mathrm{T}_{\mathrm{s}}^{\mathrm{A}, \mathrm{B}} \mathrm{x}\right\| \leqslant 2\left\|\mathrm{x}-\mathrm{T}_{\mathrm{r}}^{\mathrm{A}, \mathrm{B}} \mathrm{x}\right\|$.

Lemma 2.5 ([17, Lemma 3.3]). Let $X$ be a uniformly convex and q-uniformly smooth Banach space for some $\mathrm{q} \in(1,2]$. Assume that $\mathrm{A}$ is a single-valued $\alpha$-isa of order $\mathrm{q}$ in $\mathrm{X}$. Then, given $\mathrm{r}>0$, there exists a continuous, strictly increasing and convex function $\phi_{\mathrm{q}}: \mathrm{R}^{+} \rightarrow \mathrm{R}^{+}$with $\phi_{\mathrm{q}}(0)=0$ such that, for all $\mathrm{x}, \mathrm{y} \in \mathrm{B}_{\mathrm{r}}$,

$$
\begin{aligned}
\left\|T_{r}^{A, B} x-T_{r}^{A, B} y\right\|^{q} \leqslant & \|x-y\|^{q}-r\left(\alpha q-r^{q-1} k_{q}\right)\|A x-A y\|^{q} \\
& -\phi_{q}\left(\left\|\left(I-J_{r}^{B}\right)(I-r A) x-\left(I-J_{r}^{B}\right)(I-r A) y\right\|\right),
\end{aligned}
$$

where $\mathrm{k}_{\mathrm{q}}$ is the $\mathrm{q}$-uniform smoothness coefficient of $\mathrm{X}$.

Lemma 2.6 ([18, Lemma 3.1]). Let $\left\{a_{n}\right\}$ and $\left\{c_{n}\right\}$ are sequences of nonnegative real numbers such that

$$
a_{n+1} \leqslant\left(1-\delta_{n}\right) a_{n}+b_{n}+c_{n}, n \geqslant 1,
$$

where $\left\{\delta_{n}\right\}$ is a sequence in $(0,1)$ and $\left\{b_{n}\right\}$ is a real sequence. Assume $\sum_{n=1}^{\infty} c_{n}<\infty$. Then the following results hold:

(i) If $b_{n} \leqslant \delta_{n} M$ for some $M \geqslant 0$, then $\left\{a_{n}\right\}$ is a bounded sequence.

(ii) If $\sum_{n=1}^{\infty} \delta_{n}=\infty$ and $\limsup _{n \rightarrow \infty} \frac{b_{n}}{\delta_{n}} \leqslant 0$, then $\lim _{n \rightarrow \infty} a_{n}=0$.

By employing the technique of Maingé [19], He and Yang [15] proved the following lemma.

Lemma 2.7 ([15, Lemma 8]). Assume $\left\{s_{n}\right\}$ is a sequence of nonnegative real numbers such that

$$
s_{n+1} \leqslant\left(1-\gamma_{n}\right) s_{n}+\gamma_{n} \tau_{n}, n \geqslant 1,
$$

and

$$
s_{n+1} \leqslant s_{n}-\eta_{n}+\rho_{n}, \quad n \geqslant 1,
$$

where $\left\{\gamma_{n}\right\}$ is a sequence in $(0,1),\left\{\eta_{n}\right\}$ is a sequence of nonnegative real numbers and $\left\{\tau_{n}\right\}$, and $\left\{\rho_{n}\right\}$ are real sequences such that

(i) $\sum_{n=1}^{\infty} \gamma_{n}=\infty$

(ii) $\lim _{n \rightarrow \infty} \rho_{n}=0$.

(iii) $\lim _{k \rightarrow \infty} \eta_{n_{k}}=0$ implies $\limsup _{k \rightarrow \infty} \tau_{n_{k}} \leqslant 0$, for any subsequence $\left\{n_{k}\right\} \subset\{n\}$.

Then $\lim _{n \rightarrow \infty} s_{n}=0$.

Lemma 2.8 ([21, p. 63]). Let $\mathrm{q}>1$. Then the following inequality holds:

$$
a b \leqslant \frac{1}{q} a^{q}+\frac{q-1}{q} b^{\frac{q}{q-1}}
$$

for arbitrary positive real numbers $\mathrm{a}$ and $\mathrm{b}$. 
Lemma 2.9 ([8, Proposition 3.1]). Let $\mathrm{q}>1$ and let $\mathrm{X}$ be a real smooth Banach space with the generalized duality mapping $j_{q}$. Let $m \in \mathcal{N}$ be fixed. Let $\left\{x_{i}\right\}_{i=1}^{m} \subset X$ and $t_{i} \geqslant 0$, for all $i=1,2, \ldots, m$ with $\sum_{i=1}^{m} t_{i} \leqslant 1$. Then we have

$$
\left\|\sum_{i=1}^{m} t_{i} x_{i}\right\|^{q} \leqslant \frac{\sum_{i=1}^{m} t_{i}\left\|x_{i}\right\|^{q}}{q-(q-1) \sum_{i=1}^{m} t_{i}} .
$$

\section{Main results}

In this section, we first establish a crucial proposition and then prove our main theorem.

Proposition 3.1. Let $X$ be a uniformly convex and $q$-uniformly smooth Banach space. Let $A: X \rightarrow X$ be an $\beta$-isa of order $\mathrm{q}$ and $\mathrm{B}: \mathrm{X} \rightarrow 2^{\mathrm{X}}$ an m-accretive operator such that $\Omega:=(\mathrm{A}+\mathrm{B})^{-1}(0) \neq \emptyset$. Let $\left\{\mathrm{e}_{\mathrm{n}}\right\}$ be a sequence in $\mathrm{X}$ and $\mathrm{f}$ be a contraction on $\mathrm{X}$ with coefficient $\alpha \in[0,1)$. Let $\left\{x_{n}\right\}$ be generated by $x_{1} \in X$ and

$$
x_{n+1}=\alpha_{n} f\left(x_{n}\right)+\lambda_{n} x_{n}+\delta_{n} J_{r_{n}}^{B}\left(x_{n}-r_{n} A x_{n}\right)+e_{n}, n \geqslant 1,
$$

where $J_{r_{n}}^{B}=\left(I+r_{n} B\right)^{-1}, 0<r_{n}<\left(\beta q / k_{q}\right)^{1 /(q-1)}$ and $\left\{\alpha_{n}\right\},\left\{\lambda_{n}\right\}$, and $\left\{\delta_{n}\right\}$ are sequences in $[0,1]$ with $\alpha_{n}+\lambda_{n}+\delta_{n}=1$. If $\sum_{n=1}^{\infty}\left\|e_{n}\right\|<\infty$ or $\lim _{n \rightarrow \infty}\left\|e_{n}\right\| / \alpha_{n}=0$, then $\left\{x_{n}\right\}$ is bounded.

Proof. For each $n \in \mathcal{N}$, we put $T_{n}=J_{r_{n}}^{B}\left(I-r_{n} A\right)$ and let $\left\{y_{n}\right\}$ be defined by

$$
y_{n+1}=\alpha_{n} f\left(y_{n}\right)+\lambda_{n} y_{n}+\delta_{n} T_{n} y_{n} \text {. }
$$

Firstly, we compute the following:

$$
\begin{aligned}
\left\|x_{n+1}-y_{n+1}\right\| & =\left\|\alpha_{n}\left(f\left(x_{n}\right)-f\left(y_{n}\right)\right)+\lambda_{n}\left(x_{n}-y_{n}\right)+\delta_{n}\left(T_{n} x_{n}-T_{n} y_{n}\right)+e_{n}\right\| \\
& \leqslant \alpha_{n}\left\|f\left(x_{n}\right)-f\left(y_{n}\right)\right\|+\lambda_{n}\left\|x_{n}-y_{n}\right\|+\delta_{n}\left\|T_{n} x_{n}-T_{n} y_{n}\right\|+\left\|e_{n}\right\| \\
& \leqslant \alpha_{n} \alpha\left\|x_{n}-y_{n}\right\|+\lambda_{n}\left\|x_{n}-y_{n}\right\|+\delta_{n}\left\|x_{n}-y_{n}\right\|+\left\|e_{n}\right\| \\
& =\left(1-\alpha_{n}(1-\alpha)\right)\left\|x_{n}-y_{n}\right\|+\left\|e_{n}\right\| .
\end{aligned}
$$

By the assumptions and Lemma 2.6 (ii), we conclude that $\lim _{n \rightarrow \infty}\left\|x_{n}-y_{n}\right\|=0$. Let $z \in$ Fix $\left(T_{n}\right)$. We next show that $\left\{y_{n}\right\}$ is bounded. Indeed

$$
\begin{aligned}
\left\|y_{n+1}-z\right\| & =\left\|\alpha_{n}\left(f\left(y_{n}\right)-z\right)+\lambda_{n}\left(y_{n}-z\right)+\delta_{n}\left(T_{n} y_{n}-z\right)\right\| \\
& \leqslant \alpha_{n}\left\|f\left(y_{n}\right)-z\right\|+\lambda_{n}\left\|y_{n}-z\right\|+\delta_{n}\left\|T_{n} y_{n}-z\right\| \\
& \leqslant \alpha_{n}\left(\left\|f\left(y_{n}\right)-f(z)\right\|+\|f(z)-z\|\right)+\lambda_{n}\left\|y_{n}-z\right\|+\delta_{n}\left\|y_{n}-z\right\| \\
& \leqslant \alpha_{n} \alpha\left\|y_{n}-z\right\|+\alpha_{n}\|f(z)-z\|+\lambda_{n}\left\|y_{n}-z\right\|+\delta_{n}\left\|y_{n}-z\right\| \\
& =\left(1-\alpha_{n}(1-\alpha)\right)\left\|y_{n}-z\right\|+\alpha_{n}\|f(z)-z\| .
\end{aligned}
$$

This shows that $\left\{y_{n}\right\}$ is bounded by Lemma 2.6 (i) and hence $\left\{x_{n}\right\}$ is also bounded.

Theorem 3.2. Let $X$ be a uniformly convex and q-uniformly smooth Banach space. Let $A: X \rightarrow X$ be an $\beta$-isa of order $\mathrm{q}$ and $\mathrm{B}: \mathrm{X} \rightarrow 2^{\mathrm{X}}$ an m-accretive operator such that $\Omega:=(\mathrm{A}+\mathrm{B})^{-1}(0) \neq \emptyset$. Let $\left\{\mathrm{e}_{\mathrm{n}}\right\}$ be a sequence in $\mathrm{X}$ and $\mathrm{f}$ be a contraction on $\mathrm{X}$ with coefficient $\alpha \in[0,1)$. Let $\left\{\mathrm{x}_{\mathrm{n}}\right\}$ be generated by $\mathrm{x}_{1} \in \mathrm{X}$ and

$$
x_{n+1}=\alpha_{n} f\left(x_{n}\right)+\lambda_{n} x_{n}+\delta_{n} J_{r_{n}}^{B}\left(x_{n}-r_{n} A x_{n}\right)+e_{n}, n \geqslant 1,
$$

where $\mathrm{J}_{r_{n}}^{\mathrm{B}}=\left(\mathrm{I}+\mathrm{r}_{\mathrm{n}} \mathrm{B}\right)^{-1},\left\{\mathrm{r}_{\mathrm{n}}\right\} \subset(0, \infty)$ and $\left\{\alpha_{n}\right\},\left\{\lambda_{n}\right\}$, and $\left\{\delta_{n}\right\}$ are sequences in $[0,1]$ with $\alpha_{n}+\lambda_{n}+\delta_{n}=1$. Assume that 
(i) $\sum_{n=1}^{\infty} \alpha_{n}=\infty, \lim _{n \rightarrow \infty} \alpha_{n}=0$;

(ii) $0<\liminf _{n \rightarrow \infty} r_{n} \leqslant \limsup _{n \rightarrow \infty} r_{n}<\left(\beta q / k_{q}\right)^{1 /(q-1)}$;

(iii) $\liminf _{n \rightarrow \infty} \delta_{n}>0$;

(iv) $\sum_{n=1}^{\infty}\left\|e_{n}\right\|<\infty$ or $\lim _{n \rightarrow \infty}\left\|e_{n}\right\| / \alpha_{n}=0$.

Then $\left\{x_{n}\right\}$ strongly converges to some $z \in \Omega$.

Proof. Let $z \in \operatorname{Fix}\left(T_{n}\right)$, from Lemma 2.2 and Lemma 2.8, we have

$$
\begin{aligned}
\left\|y_{n+1}-z\right\|^{\mathrm{q}}= & \left\|\alpha_{n}\left(f\left(y_{n}\right)-z\right)+\lambda_{n}\left(y_{n}-z\right)+\delta_{n}\left(T_{n} y_{n}-z\right)\right\|^{q} \\
\leqslant & \left\|\lambda_{n}\left(y_{n}-z\right)+\delta_{n}\left(T_{n} y_{n}-z\right)\right\|^{q}+q \alpha_{n}\left\langle\left(f\left(y_{n}\right)-z\right), j_{q}\left(y_{n+1}-z\right)\right\rangle \\
\leqslant & \left\|\lambda_{n}\left(y_{n}-z\right)+\delta_{n}\left(T_{n} y_{n}-z\right)\right\|^{q} \\
& +q \alpha_{n}\left(\left\langle f\left(y_{n}\right)-f(z), j_{q}\left(y_{n+1}-z\right)\right\rangle+\left\langle f(z)-z, j_{q}\left(y_{n+1}-z\right)\right\rangle\right) \\
\leqslant & \left\|\lambda_{n}\left(y_{n}-z\right)+\delta_{n}\left(T_{n} y_{n}-z\right)\right\|^{q} \\
& \left.+q \alpha_{n} \alpha\left\|y_{n}-z\right\|\left\|y_{n+1}-z\right\|^{q-1}+q \alpha_{n}\left\langle f(z)-z, j_{q}\left(y_{n+1}-z\right)\right\rangle\right) \\
\leqslant & \| \lambda_{n}\left(y_{n}-z\right)+\delta_{n}\left(T_{n} y_{n}-z \|^{q}\right. \\
& +q \alpha_{n} \alpha\left(\frac{1}{q}\left\|y_{n}-z\right\|^{q}+\frac{q-1}{q}\left\|y_{n+1}-z\right\|^{q}\right)+q \alpha_{n}\left\langle f(z)-z, j_{q}\left(y_{n+1}-z\right)\right\rangle .
\end{aligned}
$$

After simplifying, it follows that

$$
\begin{aligned}
\left\|y_{n+1}-z\right\|^{q} \leqslant & \frac{1}{1-(q-1) \alpha_{n} \alpha}\left\|\lambda_{n}\left(y_{n}-z\right)+\delta_{n}\left(T_{n} y_{n}-z\right)\right\|^{q} \\
& +\frac{\alpha_{n} \alpha}{1-(q-1) \alpha_{n} \alpha}\left\|y_{n}-z\right\|^{q}+\frac{q \alpha_{n}}{1-(q-1) \alpha_{n} \alpha}\left\langle f(z)-z, j_{q}\left(y_{n+1}-z\right)\right\rangle .
\end{aligned}
$$

On the other hand, by Lemma 2.9 and Lemma 2.5, we obtain

$$
\begin{aligned}
\| \lambda_{n}\left(y_{n}-z\right)+ & \delta_{n}\left(T_{n} y_{n}-z\right) \|^{q} \\
\leqslant & \frac{1}{\alpha_{n} q+1-\alpha_{n}}\left(\lambda_{n}\left\|y_{n}-z\right\|^{q}+\delta_{n}\left\|T_{n} y_{n}-z\right\|^{q}\right) \\
\leqslant & \frac{1}{\alpha_{n} q+1-\alpha_{n}}\left(\lambda_{n}\left\|y_{n}-z\right\|^{q}+\delta_{n}\left(\left\|y_{n}-z\right\|^{q}-r_{n}\left(\beta q-r_{n}^{q-1} k_{q}\right)\left\|A y_{n}-A z\right\|^{q}\right.\right. \\
& \left.\left.-\phi_{q}\left(\left\|y_{n}-r_{n} A y_{n}-T_{n} y_{n}+r_{n} A z\right\|\right)\right)\right) \\
\leqslant & \frac{1-\alpha_{n}}{\alpha_{n} q+1-\alpha_{n}}\left\|y_{n}-z\right\|^{q}-\frac{\delta_{n} r_{n}\left(\beta q-r_{n}^{q-1} k_{q}\right)}{\alpha_{n} q+1-\alpha_{n}}\left\|A y_{n}-A z\right\|^{q} \\
& -\frac{\delta_{n}}{\alpha_{n} q+1-\alpha_{n}} \phi_{q}\left(\left\|y_{n}-r_{n} A y_{n}-T_{n} y_{n}+r_{n} A z\right\|\right) .
\end{aligned}
$$

By replacing (3.3) into (3.2), it follows that

$$
\begin{aligned}
\left\|y_{n+1}-z\right\|^{q} \leqslant & \left(1-\frac{\alpha_{n} q\left(1-\alpha-(q-1) \alpha_{n} \alpha\right)}{\left(1-(q-1) \alpha_{n} \alpha\right)\left(\alpha_{n} q+1-\alpha_{n}\right)}\right)\left\|y_{n}-z\right\|^{q} \\
& -\frac{\delta_{n} r_{n}\left(\beta q-r_{n}^{q-1} k_{q}\right)}{\left(1-(q-1) \alpha_{n} \alpha\right)\left(\alpha_{n} q+1-\alpha_{n}\right)}\left\|A y_{n}-A z\right\|^{q} \\
& -\frac{\delta_{n}}{\left(1-(q-1) \alpha_{n} \alpha\right)\left(\alpha_{n} q+1-\alpha_{n}\right)} \phi_{q}\left(\left\|y_{n}-r_{n} A y_{n}-T_{n} y_{n}+r_{n} A z\right\|\right) \\
& +\frac{q \alpha_{n}}{1-(q-1) \alpha_{n} \alpha}\left\langle f(z)-z, j_{q}\left(y_{n}+1-z\right)\right\rangle .
\end{aligned}
$$


We can check that $\frac{\alpha_{n} q\left(1-\alpha-(q-1) \alpha_{n} \alpha\right)}{\left(1-(q-1) \alpha_{n} \alpha\right)\left(\alpha_{n} q+1-\alpha_{n}\right)}$ is in $(0,1)$, since $1<q \leqslant 2,\left\{\alpha_{n}\right\} \subset(0,1)$ and $\lim _{n \rightarrow \infty} \alpha_{n}=0$. Moreover, by condition (ii), $\frac{\delta_{n} r_{n}\left(\beta q-r_{n}^{q-1} k_{q}\right)}{\left(1-(q-1) \alpha_{n} \alpha\right)\left(\alpha_{n} q+1-\alpha_{n}\right)}$ and $\frac{\delta_{n}}{\left(1-(q-1) \alpha_{n} \alpha\right)\left(\alpha_{n} q+1-\alpha_{n}\right)}$ are positive.

For each $n \geqslant 1$, we set

$$
\begin{aligned}
s_{n}= & \left\|y_{n}-z\right\|^{q}, \quad \gamma_{n}=\frac{\alpha_{n} q\left(1-\alpha-(q-1) \alpha_{n} \alpha\right)}{\left(1-(q-1) \alpha_{n} \alpha\right)\left(\alpha_{n} q+1-\alpha_{n}\right)}, \\
\tau_{n}= & \frac{\alpha_{n} q+1-\alpha_{n}}{1-\alpha-(q-1) \alpha_{n} \alpha}\left\langle f(z)-z, j_{q}\left(y_{n+1}-z\right)\right\rangle, \\
\eta_{n}= & \frac{\delta_{n} r_{n}\left(\beta q-r_{n}^{q-1} k_{q}\right)}{\left(1-(q-1) \alpha_{n} \alpha\right)\left(\alpha_{n} q+1-\alpha_{n}\right)}\left\|A y_{n}-A z\right\|^{q} \\
& +\frac{\delta_{n}}{\left(1-(q-1) \alpha_{n} \alpha\right)\left(\alpha_{n} q+1-\alpha_{n}\right)} \phi_{q}\left(\left\|y_{n}-r_{n} A y_{n}-T_{n} y_{n}+r_{n} A z\right\|\right), \\
\rho_{n}= & \frac{q \alpha_{n}}{1-(q-1) \alpha_{n} \alpha}\left\langle f(z)-z, j_{q}\left(y_{n+1}-z\right)\right\rangle .
\end{aligned}
$$

From (3.4), we have

$$
s_{n+1} \leqslant\left(1-\gamma_{n}\right) s_{n}+\gamma_{n} \tau_{n}, \quad n \geqslant 1,
$$

and

$$
s_{n+1} \leqslant s_{n}-\eta_{n}+\rho_{n}, \quad n \geqslant 1 .
$$

Since $\sum_{n=1}^{\infty} \alpha_{n}=\infty$, it follows that $\sum_{n=1}^{\infty} \gamma_{n}=\infty$. By the boundedness of $\left\{y_{n}\right\}$ and $\lim _{n \rightarrow \infty} \alpha_{n}=0$, we see that $\lim _{n \rightarrow \infty} \rho_{n}=0$. In order to complete the proof, by using Lemma 2.7, it remains to show that $\lim _{k \rightarrow \infty} \eta_{n_{k}}=0$ implies $\lim \sup \tau_{n_{k}} \leqslant 0$, for any subsequence $\left\{n_{k}\right\} \subset\{n\}$.

Let $\left\{n_{k}\right\}$ be a subsequence of $\{n\}$ such that $\lim _{k \rightarrow \infty} \eta_{n_{k}}=0$. So, by our assumptions and the property of $\phi_{\mathrm{q}}$, we can deduce that

$$
\lim _{k \rightarrow \infty}\left\|A y_{n_{k}}-A z\right\|=\lim _{k \rightarrow \infty}\left\|y_{n_{k}}-\gamma_{n_{k}} A y_{n_{k}}-T_{n_{k}} y_{n_{k}}+r_{n_{k}} A z\right\|=0 .
$$

This gives, by the triangle inequality, that

$$
\lim _{k \rightarrow \infty}\left\|T_{n_{k}} y_{n_{k}}-y_{n_{k}}\right\|=0 .
$$

Since $\liminf _{k \rightarrow \infty} r_{n}>0$, there is $r>0$ such that $r_{n} \geqslant r$, for all $n \geqslant 1$. In particular, $r_{n_{k}} \geqslant r$ for all $k \geqslant 1$. Lemma 2.4 (ii) yields that

$$
\left\|T_{r}^{A, B} y_{n_{k}}-y_{n_{k}}\right\| \leqslant 2\left\|T_{n_{k}} y_{n_{k}}-y_{n_{k}}\right\| .
$$

Then, by (3.5), we obtain

$$
\limsup _{k \rightarrow \infty}\left\|T_{r}^{A, B} y_{n_{k}}-y_{n_{k}}\right\| \leqslant 2 \lim _{k \rightarrow \infty}\left\|T_{n_{k}} y_{n_{k}}-y_{n_{k}}\right\|
$$

It follows that

$$
\lim _{k \rightarrow \infty}\left\|T_{r}^{A, B} y_{n_{k}}-y_{n_{k}}\right\|=0 .
$$

Let $z_{\mathrm{t}}=\mathrm{tf}\left(z_{\mathrm{t}}\right)+(1-\mathrm{t}) \mathrm{T}_{\mathrm{r}}^{\mathrm{A}, \mathrm{B}} z_{\mathrm{t}}, \mathrm{t} \in(0,1)$. By employing Lemma 2.3, we have $z_{\mathrm{t}} \rightarrow z \in \Omega$ as $\mathrm{t} \rightarrow 0$. From Lemma 2.2 we have that

$$
\begin{aligned}
\left\|z_{t}-y_{n_{k}}\right\|^{q} & =\left\|t\left(f\left(z_{t}\right)-y_{n_{k}}\right)+(1-t)\left(T_{r}^{A, B} z_{t}-y_{n_{k}}\right)\right\|^{q} \\
& \leqslant(1-t)^{q}\left\|T_{r}^{A, B} z_{t}-y_{n_{k}}\right\|^{q}+q t\left\langle f\left(z_{t}\right)-y_{n_{k}}, j_{q}\left(z_{t}-y_{n_{k}}\right)\right\rangle
\end{aligned}
$$




$$
\begin{aligned}
\leqslant & (1-t)^{q}\left(\left\|T_{r}^{A, B} z_{t}-T_{r}^{A, B} y_{n_{k}}\right\|+\left\|T_{r}^{A, B} y_{n_{k}}-y_{n_{k}}\right\|\right)^{q} \\
& +q t\left\langle f\left(z_{t}\right)-z_{t}, j_{q}\left(z_{t}-y_{n_{k}}\right)\right\rangle+q t\left\langle z_{t}-y_{n_{k}}, j_{q}\left(z_{t}-y_{n_{k}}\right)\right\rangle \\
\leqslant & (1-t)^{q}\left(\left\|z_{t}-y_{n_{k}}\right\|+\left\|T_{r}^{A, B} y_{n_{k}}-y_{n_{k}}\right\|\right)^{q} \\
& +q t\left\langle f\left(z_{t}\right)-z_{t}, j_{q}\left(z_{t}-y_{n_{k}}\right)\right\rangle+q t\left\|z_{t}-y_{n_{k}}\right\|^{q} .
\end{aligned}
$$

This shows that

$$
\left\langle z_{t}-f\left(z_{t}\right), j_{q}\left(z_{t}-y_{n_{k}}\right)\right\rangle \leqslant \frac{(1-t)^{q}}{q t}\left(\left\|z_{t}-y_{n_{k}}\right\|+\left\|T_{r}^{A, B} y_{n_{k}}-y_{n_{k}}\right\|\right)^{q}+\frac{q t-1}{q t}\left\|z_{t}-y_{n_{k}}\right\|^{q} .
$$

From (3.7) and (3.6), we obtain

$$
\begin{aligned}
\limsup _{k \rightarrow \infty}\left\langle z_{t}-f\left(z_{t}\right), j_{q}\left(z_{t}-y_{n_{k}}\right)\right\rangle & \leqslant \frac{(1-t)^{q}}{q t} M^{q}+\frac{q t-1}{q t} M^{q} \\
& =\frac{(1-t)^{q}+q t-1}{q t} M^{q}
\end{aligned}
$$

where $M=\limsup \left\|z_{t}-y_{n_{k}}\right\|, t \in(0,1)$. We see that $\frac{(1-t)^{q}+q t-1}{q t} \rightarrow 0$ as $t \rightarrow 0$. From Lemma 2.1 (ii), we know that $j_{q}$ is norm-to-norm uniformly continuous on bounded subsets of $X$. Since $z_{t} \rightarrow z$ as $t \rightarrow 0$, we have $\left\|j_{\mathrm{q}}\left(z_{\mathrm{t}}-\mathrm{y}_{\mathrm{n}_{\mathrm{k}}}\right)-\mathrm{j}_{\mathrm{q}}\left(z-\mathrm{y}_{\mathrm{n}_{\mathrm{k}}}\right)\right\| \rightarrow 0$ as $\mathrm{t} \rightarrow 0$. Observe that

$$
\begin{aligned}
& \left|\left\langle z_{\mathrm{t}}-\mathrm{f}\left(z_{\mathrm{t}}\right), j_{\mathrm{q}}\left(z_{\mathrm{t}}-y_{n_{\mathrm{k}}}\right)\right\rangle-\left\langle z-\mathrm{f}(z), j_{\mathrm{q}}\left(z-y_{n_{\mathrm{k}}}\right)\right\rangle\right| \\
& \leqslant\left|\left\langle z_{\mathrm{t}}-z+z-\mathrm{f}(z)+\mathrm{f}(z)-\mathrm{f}\left(z_{\mathrm{t}}\right), \mathrm{j}_{\mathrm{q}}\left(z_{\mathrm{t}}-y_{\mathrm{n}_{\mathrm{k}}}\right)\right\rangle-\left\langle z-\mathrm{f}(z), \mathrm{j}_{\mathrm{q}}\left(z-\mathrm{y}_{\mathrm{n}_{\mathrm{k}}}\right)\right\rangle\right| \\
& \leqslant\left|\left\langle z_{\mathrm{t}}-z, j_{\mathrm{q}}\left(z_{\mathrm{t}}-y_{n_{k}}\right)\right\rangle\right|+\left|\left\langle z-f(z), j_{\mathrm{q}}\left(z_{\mathrm{t}}-y_{n_{k}}\right)-j_{\mathrm{q}}\left(z-y_{n_{k}}\right)\right\rangle\right| \\
& +\left|\left\langle f(z)-f\left(z_{\mathrm{t}}\right), j_{\mathrm{q}}\left(z_{\mathrm{t}}-y_{n_{\mathrm{k}}}\right)\right\rangle\right| \\
& \leqslant(1+\alpha)\left\|z_{\mathrm{t}}-z\right\|\left\|z_{\mathrm{t}}-\mathrm{y}_{n_{\mathrm{k}}}\right\|^{\mathbf{q}-1}+\|z-\mathrm{f}(z)\|\left\|j_{\mathrm{q}}\left(z_{\mathrm{t}}-\mathrm{y}_{n_{\mathrm{k}}}\right)-\mathrm{j}_{\mathrm{q}}\left(z-\mathrm{y}_{\mathrm{n}_{\mathrm{k}}}\right)\right\|^{\mathbf{q}-1} .
\end{aligned}
$$

So as $t \rightarrow 0$, we get

$$
\left\langle z_{t}-f\left(z_{t}\right), j_{q}\left(z_{t}-y_{n_{k}}\right)\right\rangle \rightarrow\left\langle z-f(z), j_{q}\left(z-y_{n_{k}}\right)\right\rangle .
$$

From (3.8), as $t \rightarrow 0$, it follows that

$$
\limsup _{k \rightarrow \infty}\left\langle z-f(z), j_{q}\left(z-y_{n_{k}}\right)\right\rangle \leqslant 0 .
$$

By Proposition 3.1, $\left\{y_{n}\right\}$ is bounded, and so is $\left\{f\left(x_{n}\right)\right\}$, by condition (i) and (3.1), (3.5), we have

$$
\begin{aligned}
\left\|y_{n_{k}+1}-y_{n_{k}}\right\| & =\left\|\alpha_{n_{k}} f\left(y_{n_{k}}\right)+\lambda_{n_{k}} y_{n_{k}}+\delta_{n_{k}} T_{n_{k}} y_{n_{k}}-y_{n_{k}}\right\| \\
& \leqslant \alpha_{n_{k}}\left\|f\left(y_{n_{k}}\right)-y_{n_{k}}\right\|+\delta_{n_{k}}\left\|T_{n_{k}} y_{n_{k}}-y_{n_{k}}\right\| \rightarrow 0,
\end{aligned}
$$

as $k \rightarrow \infty$. By combining (3.9) and (3.10), we get that

$$
\limsup _{k \rightarrow \infty}\left\langle z-f(z), j_{q}\left(z-y_{n_{k}+1}\right)\right\rangle \leqslant 0
$$

It also follows that $\limsup _{k \rightarrow \infty} \tau_{n_{k}} \leqslant 0$. We conclude that $\lim _{n \rightarrow \infty} s_{n}=0$ by Lemma 2.7. Hence $y_{n} \rightarrow z$ as $\mathrm{n} \rightarrow \infty$, by Proposition 3.1, $\lim _{n \rightarrow \infty}\left\|x_{n}-y_{n}\right\|=0$, so $\lim _{n \rightarrow \infty} x_{n}=z \in \Omega$. We thus complete the proof.

By setting $\lambda_{n}=0$ for all $n \geqslant 1$, we obtain the following result:

Corollary 3.3. Let $X$ be a uniformly convex and q-uniformly smooth Banach space. Let $A: X \rightarrow X$ be an $\beta$-isa of order $\mathrm{q}$ and $\mathrm{B}: \mathrm{X} \rightarrow 2^{\mathrm{X}}$ an m-accretive operator such that $\Omega:=(\mathrm{A}+\mathrm{B})^{-1}(0) \neq \emptyset$. Let $\left\{e_{\mathrm{n}}\right\}$ be a sequence in $\mathrm{X}$ and $f$ be a contraction on $X$ with coefficient $\alpha \in[0,1)$. Let $\left\{x_{n}\right\}$ be generated by $x_{1} \in X$ and

$$
x_{n+1}=\alpha_{n} f\left(x_{n}\right)+\left(1-\alpha_{n}\right) J_{r_{n}}^{B}\left(x_{n}-r_{n} A x_{n}\right)+e_{n}, n \geqslant 1,
$$

where $\mathrm{J}_{\mathrm{r}_{n}}^{\mathrm{B}}=\left(\mathrm{I}+\mathrm{r}_{\mathrm{n}} \mathrm{B}\right)^{-1},\left\{\mathrm{r}_{\mathrm{n}}\right\} \subset(0, \infty)$ and $\left\{\alpha_{n}\right\}$ is a sequences in $[0,1]$. Assume that 
(i) $\sum_{n=1}^{\infty} \alpha_{n}=\infty, \lim _{n \rightarrow \infty} \alpha_{n}=0$;

(ii) $0<\liminf _{n \rightarrow \infty} r_{n} \leqslant \limsup _{n \rightarrow \infty} r_{n}<\left(\beta q / k_{q}\right)^{1 /(q-1)}$;

(iii) $\sum_{n=1}^{\infty}\left\|e_{n}\right\|<\infty$, or $\lim _{n \rightarrow \infty}\left\|e_{n}\right\| / \alpha_{n}=0$.

Then $\left\{x_{n}\right\}$ strongly converges to some $z \in \Omega$.

\section{Applications}

\subsection{Minimization problem}

In this subsection, we apply Theorem 3.2 to the convex minimization problem. Let $\mathrm{H}$ be a real Hilbert space. Let $F: H \rightarrow R$ be a convex smooth function and $G: H \rightarrow R$ be a convex, lower-semicontinuous and nonsmooth function. We consider the problem of finding $x^{*} \in \mathrm{H}$ such that

$$
F\left(x^{*}\right)+G\left(x^{*}\right) \leqslant F(x)+G(x),
$$

for all $x \in H$. This problem (4.1) is equivalent, by Fermat's rule, to the problem of finding $x^{*} \in H$ such that

$$
0 \in \nabla \mathrm{F}\left(x^{*}\right)+\partial \mathrm{G}\left(x^{*}\right),
$$

where $\nabla F$ is a gradient of $F$ and $\partial G$ is a subdifferential of $G$. In this point of view, we can set $A=\nabla F$ and $B=\partial G$ in Theorem 3.2. This is because if $\nabla F$ is $(1 / L)$-Lipschitz continuous, then it is L-inverse strongly monotone [2, Corollary 10]. Moreover, $\partial \mathrm{G}$ is maximal monotone [26, Theorem A]. So we obtain the following result.

Theorem 4.1. Let $\mathrm{H}$ be real Hilbert space. Let $\mathrm{F}: \mathrm{H} \rightarrow \mathrm{R}$ be a convex and differentiable function with $(1 / \mathrm{L})-$ Lipschitz continuous gradient $\nabla \mathrm{F}$ and $\mathrm{G}: \mathrm{H} \rightarrow \mathrm{R}$ be a convex and lower semi-continuous function which $\mathrm{F}+\mathrm{G}$ attains a minimizer. Let $\left\{e_{n}\right\}$ be a sequence in $\mathrm{H}$ and $\mathrm{f}$ be a contraction on $\mathrm{X}$ with coefficient $\alpha \in[0,1)$. Let $\left\{\mathrm{x}_{\mathrm{n}}\right\}$ be generated by $\mathrm{x}_{1} \in \mathrm{H}$ and

$$
x_{n+1}=\alpha_{n} f\left(x_{n}\right)+\lambda_{n} x_{n}+\delta_{n} J_{r_{n}}\left(x_{n}-r_{n} \nabla F\left(x_{n}\right)\right)+e_{n}, n \geqslant 1,
$$

where $\mathrm{J}_{r_{n}}=\left(I+r_{n} \partial G\right)^{-1},\left\{r_{n}\right\} \subset(0, \infty)$ and $\left\{\alpha_{n}\right\},\left\{\lambda_{n}\right\}$, and $\left\{\delta_{n}\right\}$ are sequences in $[0,1]$ with $\alpha_{n}+\lambda_{n}+\delta_{n}=1$. Assume that

(i) $\sum_{n=1}^{\infty} \alpha_{n}=\infty, \lim _{n \rightarrow \infty} \alpha_{n}=0$;

(ii) $0<\liminf _{n \rightarrow \infty} r_{n} \leqslant \limsup _{n \rightarrow \infty} r_{n}<2 L$;

(iii) $\liminf _{n \rightarrow \infty} \delta_{n}>0$;

(iv) $\sum_{n=1}^{\infty}\left\|e_{n}\right\|<\infty$, or $\lim _{n \rightarrow \infty}\left\|e_{n}\right\| / \alpha_{n}=0$.

Then $\left\{x_{n}\right\}$ strongly converges to a minimizer of $\mathrm{F}+\mathrm{G}$.

\subsection{Linear inverse problem}

In this subsection, we apply Theorem 3.2 to solve the unconstrained linear system

$$
\mathrm{Cx}=\mathrm{d},
$$

where $C$ is a bounded linear operator on $H$ and $d \in H$. For each $x \in H$, we define $F: H \rightarrow R$ by

$$
\mathrm{F}(\mathrm{x})=\frac{1}{2}\|\mathrm{C} x-\mathrm{d}\|^{2} \text {. }
$$

From [5] we know that $\nabla \mathrm{F}(\mathrm{x})=\mathrm{C}^{\top}(\mathrm{Cx}-\mathrm{d})$ and $\nabla \mathrm{F}$ is $\mathrm{K}$-Lipschitz continuous with $\mathrm{K}$ the largest eigenvalue 
of $C^{\top} \mathrm{C}$. So we obtain the following result.

Theorem 4.2. Let $\mathrm{H}$ be real Hilbert space. Let $\mathrm{C}: \mathrm{H} \rightarrow \mathrm{H}$ be a bounded linear operator and $\mathrm{d} \in \mathrm{H}$ with $\mathrm{K}$ the largest eigenvalue of $\mathrm{C}^{\top} \mathrm{C}$. Let $\left\{\mathrm{e}_{\mathrm{n}}\right\}$ be a sequence in $\mathrm{H}$ and $\mathrm{f}$ be a contraction on $\mathrm{X}$ with coefficient $\alpha \in[0,1)$. Let $\left\{x_{n}\right\}$ be generated by $x_{1} \in \mathrm{H}$ and

$$
x_{n+1}=\alpha_{n} f\left(x_{n}\right)+\lambda_{n} x_{n}+\delta_{n}\left(x_{n}-r_{n} C^{\top}\left(C\left(x_{n}\right)-d\right)\right)+e_{n}, \quad n \geqslant 1,
$$

where $\left\{r_{n}\right\} \subset(0, \infty)$ and $\left\{\alpha_{n}\right\},\left\{\lambda_{n}\right\}$, and $\left\{\delta_{n}\right\}$ are sequences in $[0,1]$ with $\alpha_{n}+\lambda_{n}+\delta_{n}=1$. Assume that

(i) $\sum_{n=1}^{\infty} \alpha_{n}=\infty, \quad \lim _{n \rightarrow \infty} \alpha_{n}=0$;

(ii) $0<\liminf _{n \rightarrow \infty} r_{n} \leqslant \limsup _{n \rightarrow \infty} r_{n}<2 / K$;

(iii) $\liminf _{n \rightarrow \infty} \delta_{n}>0$;

(iv) $\sum_{n=1}^{\infty}\left\|e_{n}\right\|<\infty$, or $\lim _{n \rightarrow \infty}\left\|e_{n}\right\| / \alpha_{n}=0$.

If (4.2) is consistent, then $\left\{x_{n}\right\}$ strongly converges to a solution of a linear system.

\section{Acknowledgment}

This study was supported by Scientific Research Fund of Sichuan Provincial Education Department (No.15ZA0112).

\section{References}

[1] H. Attouch, Viscosity solutions of minimization problems, SIAM J. Optim., 6 (1996), 769-806. 1

[2] J. B. Baillon, G. Haddad, Quelques proprits des oprateurs angle-borns et n-cycliquement monotones, (French) Israel J. Math., 26 (1977), 137-150. 4.1

[3] D. P. Bertsekas, J. N. Tsitsiklis, Parallel and distributed computation: numerical methods, Prentice-Hall, Englewood Cliffs, NJ, (1989); republished in 1997 by Athena Scientific, MA, (1997). 1

[4] H. Brézis, P.-L. Lions, Produits infinis de résolvantes, (French) Israel J. Math., 29 (1978), 329-345. 1

[5] C. Byrne, A unified treatment of some iterative algorithms in signal processing and image reconstruction, Inverse Problems, 20 (2004), 103-120. 4.2

[6] G. H.-G. Chen, R. T. Rockafellar, Convergence rates in forward-backward splitting, SIAM J. Optim., 7 (1997), 421-444. 1

[7] C. Chidume, Geometric properties of Banach spaces and nonlinear iterations, Lecture Notes in Mathematics, SpringerVerlag London, Ltd., London, (2009). 2.2

[8] P. Cholamjiak, A generalized forward-backward splitting method for solving quasi inclusion problems in Banach spaces, Numer. Algorithms, 71 (2016), 915-932. 1, 2.9

[9] I. Cioranescu, Geometry of Banach spaces, duality mappings and nonlinear problems, Mathematics and its Applications, Kluwer Academic Publishers Group, Dordrecht, (1990). 2.1

[10] P. L. Combettes, Iterative construction of the resolvent of a sum of maximal monotone operators, J. Convex Anal., 16 (2009), 727-748. 1

[11] P. L. Combettes, V. R. Wajs, Signal recovery by proximal forward-backward splitting, Multiscale Model. Simul., 4 (2005), 1168-1200. 1

[12] J. Douglas Jr., H. H. Rachford, On the numerical solution of heat conduction problems in two and three space variables, Trans. Amer. Math. Soc., 82 (1956), 421-439. 1

[13] J. C. Dunn, Convexity, monotonicity, and gradient processes in Hilbert space, J. Math. Anal. Appl., 53 (1976), 145-158. 1

[14] O. Güler, On the convergence of the proximal point algorithm for convex minimization, SIAM J. Control Optim., 29 (1991), 403-419. 1

[15] S.-N. He, C.-P. Yang, Solving the variational inequality problem defined on intersection of finite level sets, Abstr. Appl. Anal., 2013 (2013), 8 pages. 2, 2.7 
[16] P.-L. Lions, B. Mercier, Splitting algorithms for the sum of two nonlinear operators, SIAM J. Numer. Anal., 16 (1979), 964-979. 1

[17] G. López, V. Martín-Márquez, F.-H. Wang, H.-K. Xu, Forward-backward splitting methods for accretive operators in Banach spaces, Abstr. Appl. Anal., 2012 (2012), 25 pages. 1, 2.4, 2.5

[18] P. E. Maingé, Approximation methods for common fixed points of nonexpansive mappings in Hilbert spaces, J. Math. Anal. Appl., 325 (2007), 469-479. 2.6

[19] P. E. Maingé, Strong convergence of projected subgradient methods for nonsmooth and nonstrictly convex minimization, Set-Valued Anal., 16 (2008), 899-912. 2

[20] B. Martinet, Régularisation d'inéquations variationnelles par approximations successives, (French) Rev. Française Informat. Recherche Opérationnelle, 4 (1970), 154-158. 1

[21] D. S. Mitrinović, Analytic inequalities, In cooperation with P. M. Vasić, Die Grundlehren der mathematischen Wissenschaften, Springer-Verlag, New York-Berlin, (1970). 2.8

[22] A. Moudafi, Viscosity approximation methods for fixed-points problems, J. Math. Anal. Appl., 241 (2000), 46-55. 1

[23] G. B. Passty, Ergodic convergence to a zero of the sum of monotone operators in Hilbert space, J. Math. Anal. Appl., 72 (1979), 383-390. 1

[24] D. H. Peaceman, H. H. Rachford Jr., The numerical solution of parabolic and elliptic differential equations, J. Soc. Indust. Appl. Math., 3 (1955), 28-41. 1

[25] S. Reich, Strong convergence theorems for resolvents of accretive operators in Banach spaces, J. Math. Anal. Appl., 75 (1980), 287-292. 2.3

[26] R. T. Rockafellar, On the maximal monotonicity of subdifferential mappings, Pacific J. Math., 33 (1970), 209-216. 4.1

[27] R. T. Rockafellar, Monotone operators and the proximal point algorithm, SIAM J. Control Optim., 14 (1976), 877-898. 1

[28] W. Takahashi, N.-C. Wong, J.-C. Yao, Two generalized strong convergence theorems of Halpern's type in Hilbert spaces and applications, Taiwanese J. Math., 16 (2012), 1151-1172. 1

[29] P. Tseng, A modified forward-backward splitting method for maximal monotone mappings, SIAM J. Control Optim., 38 (2000), 431-446. 1, 1

[30] H.-K. Xu, Inequalities in Banach spaces with applications, Nonlinear Anal., 16 (1991), 1127-1138. 2

[31] H.-K. Xu, Viscosity approximation methods for nonexpansive mappings, J. Math. Anal. Appl., 298 (2004), $279-291.1$

[32] H. Zegeye, N. Shahzad, Strong convergence theorems for a common zero for a finite family of m-accretive mappings, Nonlinear Anal., 66 (2007), 1161-1169. 1 